\title{
CA19-9-producing esophageal adenocarcinoma originating from the esophageal cardia of the mid-thoracic esophagus: a case report
}

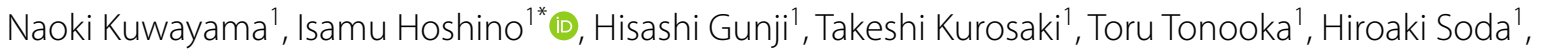 \\ Itaru Sonoda', Ryotaro Eto ${ }^{1}$, Nobuhiro Takiguchi' ${ }^{1}$, Yoshihiro Nabeya ${ }^{1}$, Makiko Itami ${ }^{2}$ and Wataru Takayama ${ }^{1}$
}

\begin{abstract}
Background: Although there are many studies on primary esophageal adenocarcinoma arising from Barrett's esophagus or ectopic gastric mucosa, reports on adenocarcinoma arising from esophageal cardiac glands are extremely rare. Herein, we report a case of mid-thoracic cancer antigen 19-9 (CA 19-9)-producing primary esophageal adenocarcinoma, which presumably originated from the cardiac glands.

Case presentation: A 74-year-old man was referred to our department with advanced esophageal cancer, which initially presented with dyspepsia. Serum levels of cancer antigen 19-9 (CA 19-9) were elevated (724.89 U/ml). Upper gastrointestinal endoscopy revealed a type 2 tumor on the posterior wall of the mid-thoracic esophagus approximately 29-32 cm from the incisor. Mucosal biopsy was consistent with a diagnosis of adenocarcinoma. Contrastenhanced computed tomography showed a circumferential wall thickening in the mid-thoracic esophagus without enlarged lymph nodes or distant metastasis. Positron emission tomography-computed tomography showed accumulation in the primary tumor, but no evidence of lymph node or distant metastasis. According to these findings, the adenocarcinoma was staged as CT3NOMO, thereby, requiring subtotal esophagectomy with lymph node dissection. Postoperative course was uneventful. Histopathologic analysis revealed a $50 \times 40 \mathrm{~mm}$ moderately differentiated adenocarcinoma with invasion to the thoracic duct and lymph node metastasis at \#108(1/4), \#109R(1/3), and \#109L(1/3). After surgery, the stage was revised to moderately differentiated pT4apN2pM0 (pStage III). Immunostaining revealed expression of CA19-9 and suggested esophageal cardiac gland origin of the tumor. Three months after the surgery, the patient showed no recurrence and is undergoing outpatient observation.
\end{abstract}

Conclusions: We experienced a case of mid-thoracic CA19-9-producing primary esophageal adenocarcinoma, which was presumed to have originated in the esophageal cardiac glands. Due to the scarcity of studies regarding this condition, specific management needs to be further clarified.

Keywords: Esophageal adenocarcinoma, Esophageal cardiac glands, CA19-9

\footnotetext{
*Correspondence: ihoshino@chiba-cc.jp

1 Division of Gastroenterological Surgery, Chiba Cancer Center, 666-2

Nitona-cho, Chuo-ku, Chiba 260-8717, Japan

Full list of author information is available at the end of the article
}

\begin{abstract}
Background
Although the incidence of esophageal adenocarcinoma has increased in the recent years with the rise of Barrett's esophageal adenocarcinoma, primary esophageal adenocarcinoma arising in the mid-thoracic esophagus, that is those not derived from Barrett's esophagus or ectopic
\end{abstract}


gastric mucosa, is extremely rare [1]. To the best of our knowledge, there has been no report of esophageal adenocarcinoma derived from esophageal cardiac glands with high CA19-9 levels. Herein, we describe a rare case of CA19-9-producing mid-thoracic esophageal adenocarcinoma, which likely originated from esophageal cardiac glands.

\section{Case presentation}

A 74-year-old man was referred to our department for advanced esophageal cancer, which initially presented with dyspepsia. Serum CA19-9 levels were elevated $(724.89 \mathrm{U} / \mathrm{ml})$. Upper gastrointestinal endoscopy revealed a type 2 tumor on the posterior wall of the mid-thoracic esophagus, approximately $29-32 \mathrm{~cm}$ from the incisor (Fig. 1a). Histopathologic analysis of the mucosal biopsy revealed adenocarcinoma. Contrastenhanced computed tomography showed circumferential wall thickening in the mid-thoracic esophagus without enlarged lymph nodes and distant metastasis (Fig. 1b, c). Positron emission tomography-computed tomography showed accumulation in the primary tumor, but no evidence of lymph node or distant metastasis (Fig. 1d). Accordingly, a clinical stage of cT3NOM0 was established, thereby, requiring subtotal esophagectomy with lymph node dissection. The postoperative course was uneventful. Histopathologic analysis revealed a $50 \times 40 \mathrm{~mm}$ moderately differentiated adenocarcinoma with invasion in the thoracic duct (pT4a) and lymph node metastasis at \#108(1/4), \#109R(1/3), and \#109L(1/3) (pN2). These postoperative findings warranted the revision of the diagnosis and stage to moderately differentiated adenocarcinoma pT4apN2pM0 (pStage III) (Figs. 2 and 3a).

Immunostaining revealed the increased diffuse expression of CA19-9 in the main tumor and all three metastatic lymph nodes, suggesting that it was a CA19-9-producing tumor. Moreover, it was also positive for (1) MUC5AC, epithelial-type mucus core proteins of the gastric gland fossa and (2) MUC6, a glandular-type mucus core protein present in the pyloric and cardiac glands (Fig. 3c, d). Furthermore, histopathologically, no ectopic gastric mucosa was found in the background mucosa. The absence of

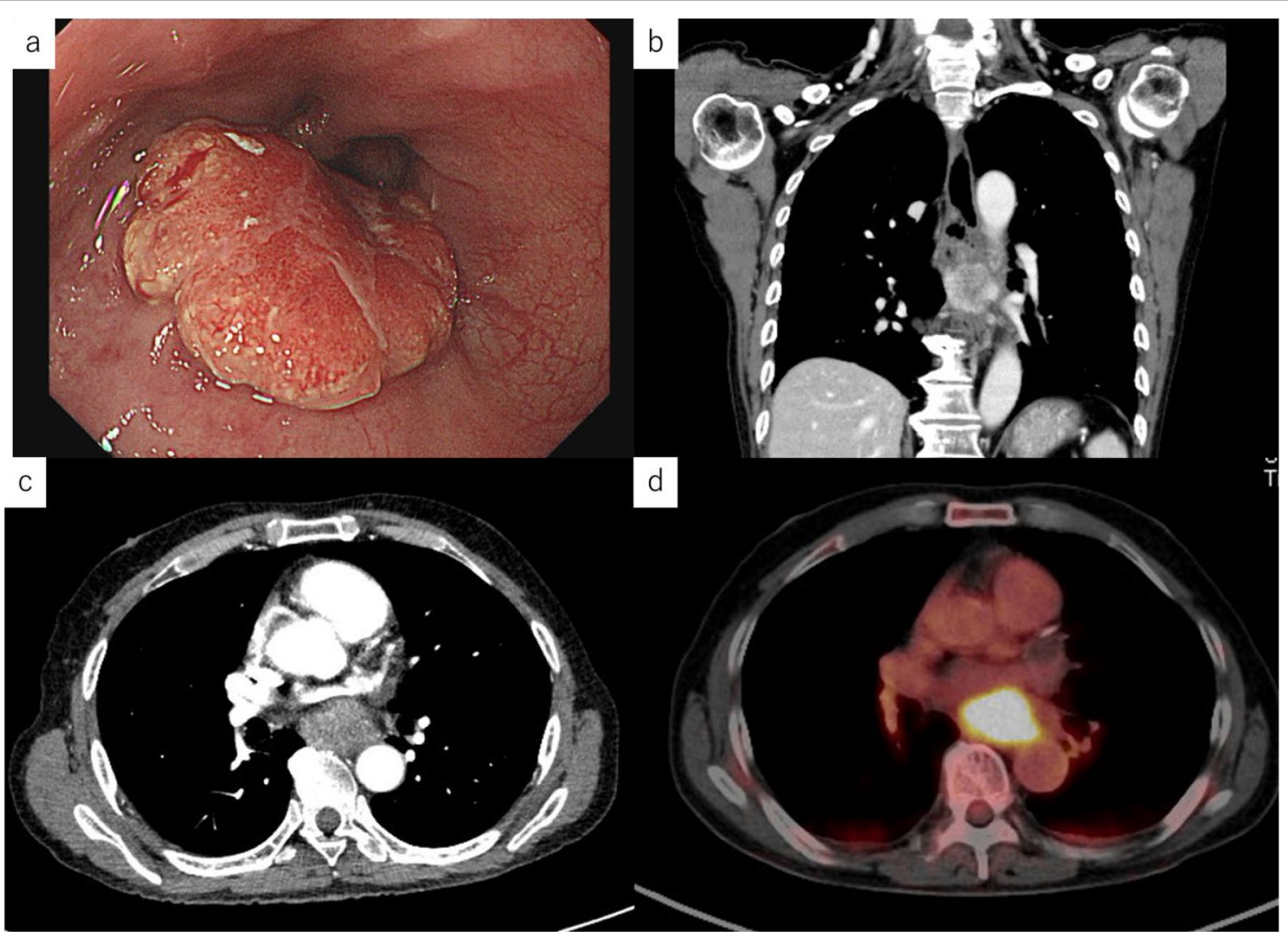

Fig. 1 Test results of the patient. a Upper gastrointestinal endoscopy showing a type 2 tumor on the posterior wall of the mid-thoracic esophagus, approximately 29-32 cm from the incisor. b, c Contrast-enhanced computed tomography revealing a circumferential wall thickening in the mid-thoracic esophagus without enlarged lymph nodes or distant metastasis. $\mathbf{d}$ Positron emission tomography-computed tomography showing accumulation in the primary tumor, but no evidence of lymph node metastasis or distant metastasis 


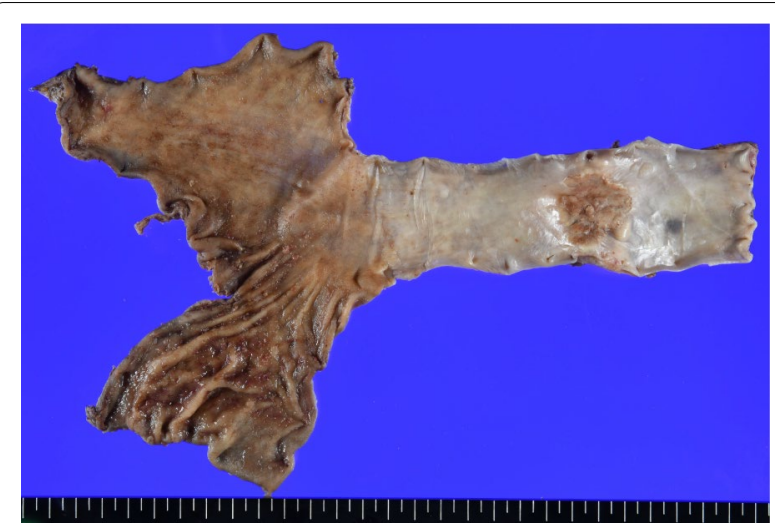

Fig. 2 Histopathologic analysis revealed a $50 \times 40 \mathrm{~mm}$ moderately differentiated adenocarcinoma with invasion to the thoracic duct and lymph node metastasis at \#108(1/4), \#109R(1/3), and \#109L(1/3). After surgery, the stage was revised to moderately differentiated pT4apN2pMO (pStage III)
Barrett's epithelium and ectopic gastric mucosa in the mid-thoracic region led us to speculate that the tumor originated from the esophageal cardia. Three months postoperatively, the patient showed no recurrence and is undergoing outpatient observation.

\section{Discussion}

Primary esophageal adenocarcinoma is thought to originate from four sites: (1) esophageal glands proper, (2) esophageal cardiac glands, (3) ectopic gastric mucosa, and (4) Barrett's esophagus [1,2]. The number of reports on esophageal adenocarcinoma has increased in the recent years, most of which are arising from Barrett's esophagus. Mid-thoracic primary esophageal adenocarcinoma, which is not arising from Barrett's esophagus or ectopic gastric mucosa, is an extremely rare disease. To the best of our knowledge, there has been no report of primary esophageal adenocarcinoma originating from the esophageal cardiac glands in the mid-thoracic region.

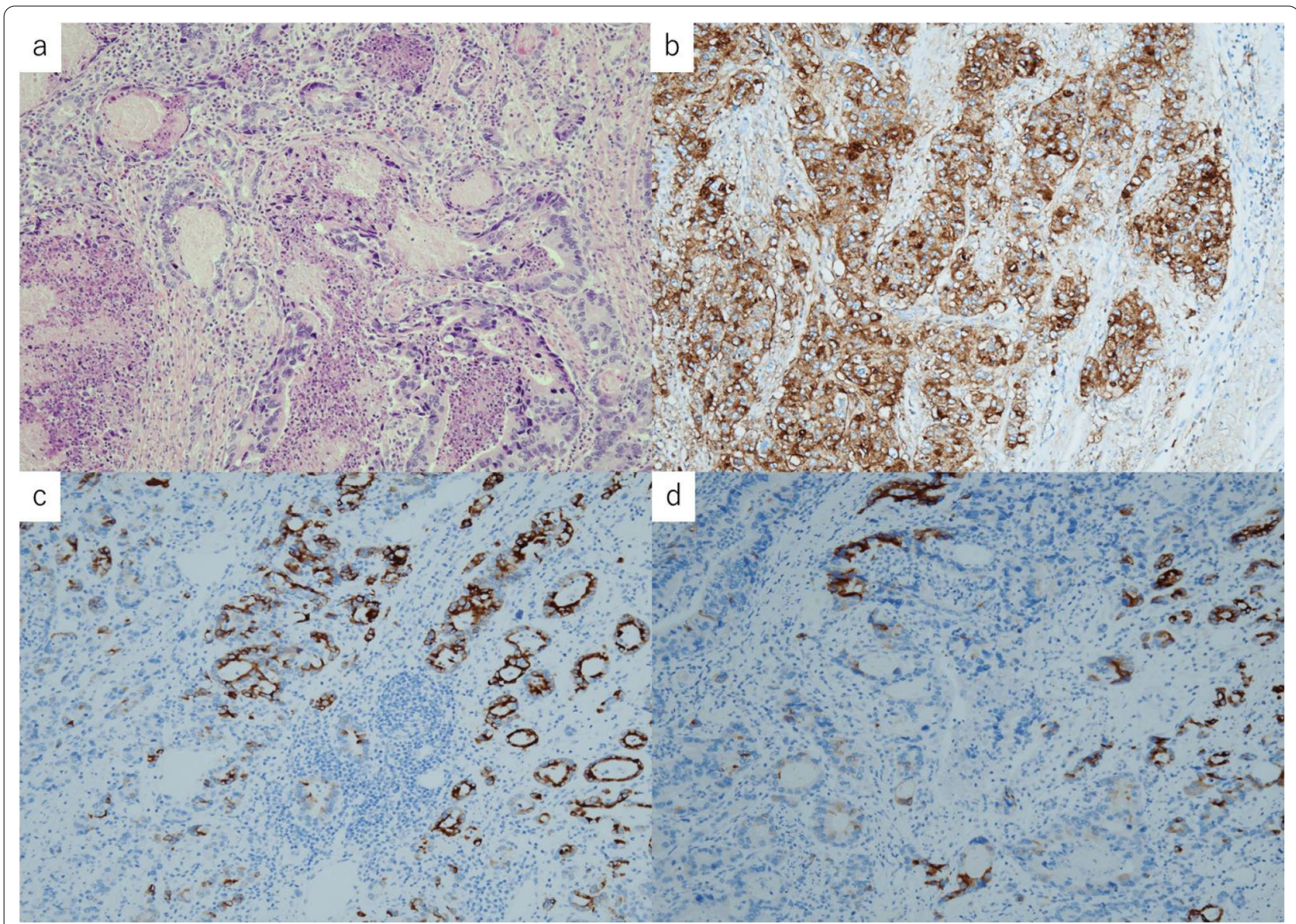

Fig. 3 Histopathologic and immunostaining analysis. a Histopathologic analysis revealed a $50 \times 40 \mathrm{~mm}$ moderately differentiated adenocarcinoma. b Immunostained sections showing increased diffuse expression of CA19-9 in the main tumor and in all three metastatic lymph nodes. $\mathbf{c}$, d Immunostaining was also positive for MUC5AC (c), epithelial-type mucus core proteins of the gastric gland fossa, and MUC6 (d), a glandular-type mucus core protein present in the pyloric and cardiac glands 
Esophageal cardiac glands are mucous glands found in the intrinsic layer of the esophageal mucosa, usually covered by superficial squamous epithelium [3]. The average width of these gland based on histopathologic examinations is around $4 \mathrm{~mm}(1-26 \mathrm{~mm})$. This is continuously distributed from the gastric to the esophageal side. However, in cases of gastroesophageal reflux disease or short-segment Barrett's esophagus, the glands are more extensive, reaching up to the squamous surface and are sometimes discontinuous $[4,5]$. As a morphologic feature of the tumor, the esophageal cardiac glands are located in the intrinsic layer of the mucosa. In contrast, esophageal glands originate from the submucosal tissue. It has been reported that tumors derived from esophageal glands tend to be more deeply invasive such that these often develop in a submucosal tumor-like fashion [6]. In the present case, it was difficult to infer the origin of the tumor based on the morphologic characteristics of the tumor alone. However, immunohistochemical analysis had aided in the establishment of its etiology. Results revealed that the primary tumor had increased diffuse expression of MUC5AC and MUC6. MUC5AC is gastric epithelial-type mucus core protein found in the surface epithelium of normal gastric mucosa. In contrast, MUC6 is a pyloric gland-type mucus core protein found in pyloric and cardiac glands. These MUCs are usually absent in esophageal intrinsic glands [7, 8]. Moreover, the absence of Barrett's epithelium and the rare occurrence of ectopic gastric mucosa in the mid-thoracic region led us to speculate that the tumor originated from the esophageal cardia [9].

In addition, due to the increased preoperative serum CA19-9 levels and the positive CA19-9 expression in the tumor and metastatic lymph nodes during immunostaining, this tumor was suspected to be a CA19-9-producing tumor. Although CA19-9 is a widely used tumor marker for gastrointestinal cancers (for example, gastric, colorectal, pancreatic, and biliary cancers), its exact significance in esophageal adenocarcinoma has not been clarified [10]. Tokunaga et al. found that for adenocarcinoma of the esophagogastric junction, carcinoembryonic antigen (CEA) and CA19-9 positivity rates were significantly higher $(P=0.002$ and $<0.001$, respectively $)$ in patients with myometrial and deeper tumor invasion $(P \leq 0.0063$ $[=0.05 / 8])$. Moreover, CA19-9 was associated with higher cancer-specific survival (multivariate hazard ratio $[\mathrm{HR}]=3.89$, 95\% confidence interval $[\mathrm{CI}] 1.41-10.33$; $P=0.010$ ) and overall survival (multivariate $\mathrm{HR}=2.43$, 95\% CI $1.03-5.35 ; P=0.043$ ) as independent prognostic factors [11]. Scarpa et al. reported that serum levels of CA19-9 and CEA were significantly higher in patients with unresectable esophageal adenocarcinoma compared to those with resectable esophageal adenocarcinoma
( $P=0.001$ and $P=0.003$, respectively), indicating that these were significant biomarkers of highly advanced cancer [12]. Van der Kaaij et al. found that median overall survival was different among patients with normal levels of both CEA and CA19-9 ( $n=59,51$ months), elevated levels of CA19-9 only ( $n=19,24$ months), and elevated levels of both CEA and CA19-9 ( $n=11,11$ months) $(P<0.001)$. They reported that the median time to tumor recurrence was 34 months in patients with normal CEA and CA19-9 levels and 7 months in patients with elevated levels $(P=0.003)[13]$.

Although further prospective validation is needed, CA19-9 is a useful marker for estimating the progression and prognosis of patients with esophageal adenocarcinoma. Together with other clinical information, it has the potential for identifying patients who will likely benefit from early systemic treatment. Regarding the choice of treatment for the patient, we chose upfront surgery for a preoperative diagnosis of cT3N0M0. This was considered because of the patient's relatively old age, difficulty in oral intake, and significant weight loss, which made it difficult to complete the preoperative treatment. In general, the efficacy of preoperative chemotherapy and chemoradiation has been reported for both squamous cell carcinoma and adenocarcinoma in locally advanced esophageal cancer without distant metastasis and those with nodal metastasis $[14,15]$.

Recently, CA19-9 has been found to be specifically elevated in pancreatic cancer recurrence and is expected to be useful for surveillance of adenocarcinoma [16].

In this case, CA19-9 levels remained high (647.49 U/ $\mathrm{ml}$ ) after surgery. It is reported that CA19-9 is an independent prognostic factor in gastric cancer [17], and that patients whose CA19-9 does not normalize after resection have a poor prognosis [18]. There are no reports on the usefulness of CA19-9 for postoperative surveillance of esophageal adenocarcinoma, and further accumulation of cases is needed. Thus, careful follow-up is necessary in this case as well.

\section{Conclusions}

In conclusion, most of the previous reports have focused on esophageal adenocarcinoma derived from Barrett's esophagus in various locations (lower thoracic, abdominal, and junctional). We experienced a case of CA199-producing primary esophageal adenocarcinoma, which was presumed to have originated from the esophageal cardiac glands of the mid-thoracic esophagus. Due to the scarcity of studies regarding CA19-9-producing adenocarcinoma derived from the cardiac glands in the mid-thoracic esophagus, the specific treatment is still unknown. Further studies are crucial for establishing safe and effective approaches for adequate management. 


\section{Abbreviations}

CA 19-9: Cancer antigen 19-9; CEA: Carcinoembryonic antigen; HR: Hazard ratio; $\mathrm{Cl}$ : Confidence interval.

\section{Acknowledgements}

None.

\section{Authors' contributions}

IH made substantial contributions to the concept and design of the case report. NK, IH, HG, TT, HS, IS, RE, NT, YN and MI envisioned the study, participated in its design and coordination, and helped draft the manuscript. The authors have read and approved the final manuscript.

\section{Funding}

None of the authors received any funding for this study.

\section{Availability of data and materials}

Data sharing is not applicable to this article, as no data sets were generated or analyzed during the study.

\section{Declarations}

Ethics approval and consent to participate

This study was approved by the Chiba Cancer Center ethical committee

\section{Consent for publication}

Written informed consent was obtained from the patient for publication of this case report and accompanying images.

\section{Competing interests}

The authors declare no competing interests.

\section{Author details}

${ }^{1}$ Division of Gastroenterological Surgery, Chiba Cancer Center, 666-2 Nitona-cho, Chuo-ku, Chiba 260-8717, Japan. ${ }^{2}$ Division of Surgical Pathology, Chiba Cancer Center, 666-2 Nitona-cho, Chuo-ku, Chiba 260-8717, Japan.

Received: 19 May 2021 Accepted: 9 July 2021

Published online: 15 July 2021

\section{References}

1. Azzopardi JG, Menzies T. Primary oesophageal adenocarcinoma. Confirmation of its existence by the finding of mucous gland tumours. $\mathrm{Br} J$ Surg. 1962;49:497-506.

2. Endoh Y, Miyawaki M, Tamura G, Watanabe H, Motoyama T. Esophageal adenocarcinoma that probably originated in the esophageal gland duct: a case report. Pathol Int. 1999;49(2):156-9. https://doi.org/10.1046/j.14401827.1999.00838.x.

3. Lagergren J, Lagergren P. Recent developments in esophaegeal adenocarcinoma. CA Cancer J Clin. 2013;63:232-48.

4. Nakanishi Y, Saka M, Eguchi T, et al. Distribution and significance of the oesophageal and gastric cardiac mucosae: a study of 131 operation specimens. Histopathology. 2007;51:515-9.
5. Sarbia M, Donner A, Gabbert HE. Histopathology of the gastroesophageal junction; a study on 36 operation specimens. Am J Surg Pathol. 2002;26:1207-12.

6. Yoshida M, Ide H, Yamada A, et al. Early detection of adenocarcinoma of the esophagus. Endoscopy. 1986:18:44-8.

7. Machado JC, Nogueira AM, Carneiro F, Reis CA, Sobrinho-Simões M. Gastric carcinoma exhibits distinct types of cell differentiation: an immunohistochemical study of trefoil peptides (TFF1 and TFF2) and mucins (MUC1, MUC2, MUC5AC, and MUC6). J Pathol. 2000;190(4):437-43.

8. Arul GS, Moorghen M, Myerscough N, Alderson DA, Spicer RD, Corfield AP. Mucin gene expression in Barrett's oesophagus: an in situ hybridisation and immunohistochemical study. Gut. 2000;47(6):753-61. https:// doi.org/10.1136/gut.47.6.753.

9. Akanuma N, Hoshino l, et al. Primary esophageal adenocarcinoma arising from heterotopic gastric mucosa: report of a case. Surg Today. 2013;43(4):446-51. https://doi.org/10.1007/s00595-012-0206-9.

10. Acharya A, Markar SR, Matar M, et al. Use of tumor markers in gastrointestinal cancers: surgeon perceptions and cost-benefit tradeoff analysis. Ann Surg Oncol. 2017;24:1165-73.

11. Tokunaga R, Imamura Y, Nakamura K, Uchihara T, et al. Carbohydrate antigen 19-9 is a useful prognostic marker in esophagogastric junction adenocarcinoma. Cancer Med. 2015;4(11):1659-66. https://doi.org/10. 1002/cam4.514.

12. Scarpa M, Noaro G, Saadeh L, Cavallin F, Cagol M, Alfieri R, Plebani M, Castoro C. Esophageal cancer management: preoperative CA19.9 and CEA serum levels may identify occult advanced adenocarcinoma. World J Surg. 2015;39(2):424-32. https://doi.org/10.1007/s00268-014-2835-1.

13. van der Kaaij RT, Voncken FEM, van Dieren JM, Snaebjornsson P, Korse CM, Grootscholten C, Aleman BMP, van Sandick JW. Elevated pretreatment CEA and CA19-9 levels are related to early treatment failure in esophageal adenocarcinoma. Am J Clin Oncol. 2019;42(4):345-50. https://doi. org/10.1097/COC.0000000000000525.

14. TepPer J, Krasna MJ, Niedzwiecki D, et al. Phase III trial of trimodality therapy with cisplatin, fluorouracil, radiotherapy, and surgery compared with surgery alone for esophageal cancer: CALGB 9781. J Clin Oncol. 2008;26:1086-92.

15. Shapiro J, et al. Neoadjuvant chemoradiotherapy plus surgery versus surgery alone for oesophageal or junctional cancer (CROSS): long-term results of a randomised controlled trial. Lancet Oncol. 2015;16(9):1090-8.

16. Rieser CJ, Zenati M, Hamad A, Al Abbas Al, Bahary N, Zureikat AH, Zeh HJ 3rd, Hogg ME. CA19-9 on postoperative surveillance in pancreatic ductal adenocarcinoma: predicting recurrence and changing prognosis over time. Ann Surg Oncol. 2018;25(12):3483-91. https://doi.org/10.1245/ s10434-018-6521-7.

17. Kim JH, Jun KH, Jung H, Park IS, Chin HM. Prognostic value of preoperative serum levels of five tumor markers (carcinoembryonic antigen, CA19-9, alpha-fetoprotein, CA72-4, and CA125) in gastric cancer. Hepatogastroenterology. 2014;61(131):863-9.

18. Kwon OK, Yu W, Chung H. Prognostic value of postoperative CA19-9 normalization in patients with advanced gastric cancer. Hepatogastroenterology. 2013;60(122):240-3. https://doi.org/10.5754/hge12585.

\section{Publisher's Note}

Springer Nature remains neutral with regard to jurisdictional claims in published maps and institutional affiliations. 